\title{
Heuristic and Optimized Sensor Tasking Observation Strategies with Exemplification for Geosynchronous Objects
}

\author{
Carolin Frueh ${ }^{1}$ \\ Purdue University, West-Lafayette, IN, USA \\ Hauke Fielder ${ }^{2}$ and Johannes Herzog ${ }^{3}$ \\ Deutsches Zentrum für Luft- und Raumfahrt ((DLR), German Aerospace Center), Germany
}

With the new space fence technology, the catalog of known space objects is expected to increase to the order of 100'000 objects. Objects need to be initially detected, and sufficient observations need to be collected to allow for a first orbit determination. Furthermore, the objects have to be re-observed regularly, to keep them in the catalog, as the position uncertainty of the objects increases over time, due to unmodeled dynamic effects. Only a small number of ground-based and even fewer space-based sensors are currently available that are able to collect observations, compared to the large number of objects that need to be observed. This makes efficient sensor tasking, that takes into account the realistic ramifications of the problem, crucial in building up and maintaining a precise and accurate catalog of space objects. The time-varying sensor performance and specific sensor constraints are influenced by the sensor location and observational environmental effects, sensor hardware, processing software and observation modes. This paper shows a new method of solving sensor tasking as an optimization problem translating the heuristic principles that have been successfully applied in sensor tasking of actual SSA networks in a rigorous mathematical framework. A computationally fast near optimal solution is presented, outperforming traditional heuristic sensor tasking methods. Applications of the methodology are shown via the example of the geosynchronous objects listed in the USSTRATCOM two-line element catalog. The results are compared to state of the art observation strategies.

\section{Introduction}

One of the aims of Space Situational Awareness (SSA) and a prerequisite for Space Traffic Management (STM) is the build-up and maintenance of an as complete and as comprehensive as possible information on each individual space object. Space objects are objects of human or natural origin, which reside in the near-earth region. The set of information on the objects is often referred to as a catalog; a catalog can include a plethora of information; a minimum is the dynamical information, such as the state, position, and velocity or equivalent, and some characterization of the associated uncertainty; the latter is often called state probability density function (pdf). Additional information can include object characterization information,

\footnotetext{
${ }^{1}$ Assistant Professor, School of Aeronautics and Astronautics, Purdue University, West-Lafayette, IN, USA, cfrueh@purdue.edu

2 Section Lead Space Situational Awareness, Deutsches Zentrum für Luft- und Raumfahrt ((DLR), German Aerospace Center), German Space Operations Center (GSOC), Wessling, Germany, Hauke.Fiedler@dlr.de

${ }^{3}$ Researcher, Deutsches Zentrum für Luft- und Raumfahrt ((DLR), German Aerospace Center), German Space Operations Center (GSOC), Wessling, Germany, Johannes.Herzog@dlr.de
} 
such as shape, attitude, materials or operational state.

Information can be collected either via direct communication with the operational human-made space assets. However, only a minority of the human-made objects are active, and of course communication is limited to the owner-operator; furthermore, direct communication does not automatically guarantee a good determination of the satellite state probability density function, as communication pointing does not have to be targeted. Information of uncooperative targets can be gained with active and passive ground-based or space-based sensors. The expected number of targets is in the order of 100'000 objects of interest [26]. In comparison to this number, the number of sensors to collect independent information is relatively small. The problem is exacerbated by the fact that often only a few or a single object is observable in the field of view at a time. The large relative velocity differences between sensors and objects in different orbital regions prevent the simultaneous detection of very diverse objects, even if the field of view of the sensor would allow for it. In general, larger fields of view of the sensor leads to a diminished accuracy of the measurements. The forces acting upon the object can only be modeled to a limited accuracy. However, even with perfect knowledge of the dynamics, the non-linear nature of the problem, leads to an increasing position uncertainty. This makes re-observation of the objects necessary to keep custody. Highest accuracies well below the arcsecond level are needed for successful use of SSA results in Collision Avoidance (CA).

Well-designed sensor tasking is hence crucial. Traditionally, so-called surveys and follow-up observations are discriminated. Traditionally, survey and follow-up strategies have been developed for geosynchronous orbital regions [1, 23, 24, 27, 28]. Similar strategies have been adapted for the low Earth region for radar sensors [4]. In surveys are used for the detection of new objects, for which no a priori information is available. Parts of the sky are scanned systematically. Follow-up observations are scheduled using orbital information, to improve the orbital information; the telescope is pointed to the predicted astrometric position of the object, respectively, the highest probability region of the projected pdf. Catalog build-up, and maintenance can be done with a survey only or a combination of survey and follow-up observations.

In recent research, these classical methods have been contrasted with other sensor tasking strategies. In early work, [31] information theoretic measures of Fisher Information gain in combination with Lyapunov exponents have been utilized. In recent work, Fujimoto and Nafi [25] investigated different cost function models to evaluated different sensor tasking scenarios, based upon the traditional stripe scanning. Some other strategies are focusing on combining finite set statistics with sensor tasking [5]. Utilizing a CPHD approach, the decision of $[13,14]$ investigates the RÃl'nyi and Cauchy-Schwarz measures for the sensor tasking. Whereas the former investigates object uncertainties that might be only partially in the field of view, [22] addresses the problem when initial uncertainty is much larger than the available field of view. In [16], well known Dempster-Shafer theory has been used to solve the sensor tasking problem. [17][18] use a more generic cost function, however focus on the aspect of deep reinforcement learning to find an optimal sensor tasking solution.

The Deutsches Zentrum für Luft- und Raumfahrt (DLR, German Aerospace Center) is currently in the process of building a network of small robotic wide-field telescopes, the Small Aperture Robotic Telescope network (SMARTnet), with several observation locations around the globe. The aim is to build and maintain a complete comprehensive coverage of the geosynchronous region and to build and maintain a catalog of the full probability density function of all detectable space objects. Maintaining a catalog refers to sustaining uncertainty levels that are low enough to allow for unique identification of objects via catalog 
matching and efficient collision avoidance. The development of adequate survey and follow-up strategies are an integral part of the development and operation of SMARTnet. In the current investigation, the focus is laid on the first of the operational stations of SMARTnet, located in Zimmerwald, Switzerland.

In this paper, a new way of planning and conducting surveys is presented, in treating the sensor tasking and object coverage as an optimization problem with via a weighted sky area approach in combination with the diversification of the optimization scheme. The benefit of this paper is that it provides the setup for a methodology that can operate in the presence or absence of prior information and fuses principles that are known from heuristic sensor tasking into a rigorous mathematical scheme. In the course of the paper the optimization scheme is approximated with a near-optimal formulation and greedy solution mechanism, allowing for instant computation of the sensor tasking scheme. The paper is organized as the following: In the first section, classical survey strategies are illustrated. In the second section, the new approach is introduced. In the third section, the near-optimal computationally fast solution to the optimization problem is presented. In section four, simulation results are shown for surveys of the geosynchronous region. A direct comparison with classical stripe scanning methods is made. The paper concludes with a summary and conclusions. Preliminary research on this topic has been published in [9] and[10]. A follow-up paper uses the framework presented in this paper and shows its usability in the absence of prior object specific information [11]. The framework has also been successfully applied to space-based scenarios, and different optimization algorithms have been evaluated [19], showing that the fast algorithm, presented in this paper, although sub-optimal is performing well.

\section{Classical Surveys}

In classical surveys for geosynchronous objects, so-called declination stripes are scanned [1, 6, 15, 24, 27-29]. These stripes are formed by keeping a fixed right ascension and visiting several declinations. The declinations are chosen as such that the center of the pointing direction is placed at the declination, which is one field of view width higher than the previous declination. Often a small overlap is used to in order not to miss objects, which are just at the edge of the field of view, computing the new declination as the width of the field of view minus this overlap compared to the previous declination. A number of $l$ exposures are made for each of the $h$ different declinations that are visited in a stripe. Then after $h \cdot l$ number of exposures, the first declination is visited again. A pictograph illustrating one stripe is shown in Fig.8. This means, per object a so-called tracklet, a set of at maximum $l$ densely spaced observations, is formed. Because the time between the observations is small, they basically amount to one observation in an astrodynamic sense. The formation of tracklets in contrast to single observations is nevertheless advantageous: Besides cosmic ray and spurious detection rejection on the single frame, which has severe limitations, cosmic ray and wrong detection rejection can be significantly increased, when tracklets are formed; with moderate conservative tuning nearly 100 percent true detections may be reached [12]. Secondly, besides the astrometric position of a single detection, astrometric velocity information may be extracted with lower uncertainty, when forming tracklets: As long as the rate is nearly constant over the observation time, $n$ samples are available to find average rates, being superior over a single sample. Rate extraction on single detections is only possible, either when the object is not tracked and via measuring the streak direction and length induced by the apparent relative motion. This, however, has the effect that the signal of the object is smeared over several pixels on purpose, leading to a reduced signal to noise, which reduces the accuracy to detect beginning and end of the streak or even leads to disintegrated streaks []. If the object is tracked, the only the tracking velocity of the mechanical system or the pre-computed ephemeris is available. In the first case, it is well established that the 


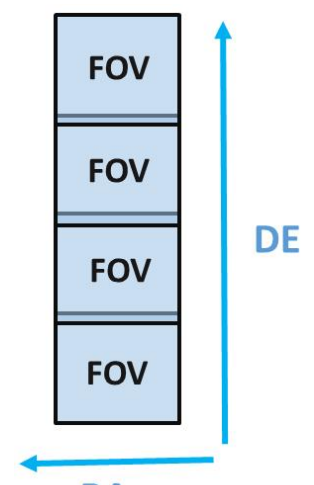

RA

Fig. 1: Illustration of the formation of declination (DE) stripes for a given field of view (FOV); right ascension (RA) is kept fixed within the stripe.

mechanical system is inferior to the extraction of pixel positions, and timing in an operational system, in the latter no actual observation update has taken place. Tracklets also allow already a reliable catalog correlation on the single tracklet level, whereas catalog correlation is often ambiguous on a single measurement level [12].

\section{A. One-Stripe strategy}

In the one stripe strategy, one declination stripe is scanned, leading to one tracklet per object within one observation night, for the geosynchronous orbital region. An important factor herewith is the cycle time, that is the time, which it takes until the first declination is used again. The cycle time is given by:

$$
t_{\text {cycle,one }}=h\left(l \cdot t_{\exp }+(l-1) t_{\text {readout }}\right)+(h-1) \cdot t_{1}+t_{2},
$$

where $t_{\exp }$ is the exposure time, $t_{\text {readout }}$ is the readout time of the image, $t_{1}$ is either the time to reposition the telescope within the stripe or the readout time, whichever is the larger of the two. Both processes can be done in parallel. $t_{2}$ is either the repositioning time of the telescope to the initial declination position or the readout time again, with the same argumentation as before. Depending on the telescope setup both times can be identical, as repositioning is dominated by the vibration settle time of the telescope mount rather than slewing time. In general either repositioning time is larger than the readout time.

To not miss an object that is passing through the declination stripe during the time the stripe is scanned, the stripe has to be leak proof. This means the cycle time $t_{c y c l e}$ needs to be shorter than the time it takes one of the target objects to pass through the field of view (FOV). The passing time is:

$$
T_{\text {pass }}=\frac{d_{\mathrm{FOV}}}{\left|\boldsymbol{v}_{\mathrm{obj}}\right|},
$$

assuming $d_{\mathrm{FOV}}$ is the distance the object can travel within the field of view for the given object velocity $v_{\text {obj }}$ of the object. For perfectly geosynchronous objects it amounts to:

$$
v_{\mathrm{obj}, \mathrm{GEO}}=\frac{2 \pi}{24 \mathrm{~h}} .
$$




\section{B. Two-Stripe Strategy}

For a successful first orbit determination, at least two observations spaced significantly in anomaly are needed. This is aimed for with the two stripe strategy for the geosynchronous region. Only after the last declination of this second stripe is scanned, the telescope is repositioned to the first declination of the first stripe. This increases the cycle to:

$$
t_{\text {cycle }, \mathrm{two}}=\sum_{j=1,2} h_{j}\left(l_{j} \cdot t_{\mathrm{exp}, j}+\left(l_{j}-1\right) t_{\mathrm{readout}}\right)+\left(h_{j}-1\right) \cdot t_{\mathrm{repos} 1, \mathrm{j}}+\tilde{t}_{\mathrm{repos}, \mathrm{j}}
$$

most often the number of exposures, the exposure times and declination directions and declination repositioning times are the same for both stripes. $\tilde{t}_{\text {repos } 2, \mathrm{j}}$ is the repositioning time from the final declination of one stripe to the first declination of the other stripe. It is possible that the repositioning time from stripe one to stripe two differs from the time to perform the reverse.

Several objectives can be leveraged in selecting the position of the two stripes; usually, two are dominating. One objective for selecting the relative positioning of the stripes is to obtain two observation tracklets per detected objects within the same night. Two observations with a significant anomaly difference are advantageous for a successful first orbit determination without a priori knowledge. The spacing of the stripes is then balanced with the cycle time and the duration of the night. Often a simple criterion on spacing the two stripes by one hour in right ascension is adopted to meet the objective. This means, the second stripe is started one hour after the first one, and observing both in parallel, until one hour prior to the end of the night. By this selection scheme, in general, two observations are gained, spaced by 15 degrees in anomaly for geosynchronous objects, as long as the object passes through the declinations that are covered by both stripes. That the objects pass through both stripes is equivalent to a limitation on orbital planes that are observed and covered. Besides these obvious advantages, this selection also bears several disadvantages. First, the scenario can be thwarted by the earth's shadow. It also does not provide an optimal spacing in anomaly, the selection of one hour is arbitrary, balancing empirically the anomaly spacing with the chances to gain two observations within the same night in combination with the coverage of orbital planes. Furthermore, illumination conditions are not taken into account.

A second objective that is often chosen for the placing of the two stripes is placing the stripes to minimize the phase angle under which the objects are observed. The phase angle is defined as the angle formed by the three points observer, object, and sun, with the vertex residing in the object position and the two legs being formed by the direction from the object to the observer and from the object to the sun. The underlying assumption is to provide for the best possible illumination of the objects, and hence increasing the probability of detection. Indeed, for spherical objects with uniform reflection properties, the most light is reflected towards the observer at the smallest possible phase angle at which the object is still illuminated (not in earth shadow). The same is true for satellites that align large flat Lambertian surfaces towards the sun; an example are solar panels that are directed towards the sun for optimal energy efficiency for the onboard satellite electronics. Solar panels have a Lambertian but also a specular reflection component. Flat surfaces that have a specular component produce glints, specular reflections, that are usually brighter than the Lambertian reflection. However, exact glint conditions within 0.5 degrees have to be fulfilled. Albeit, glints off the solar panels do occur at the small phase angle conditions, they are in no way guaranteed by the small phase angle condition alone and are only caught by chance without precise knowledge of the satellite geometry. Glints from the antenna and other satellite parts occur; the small phase angle condition at Earth shadow is not relevant for those.

In the simplification assuming spherical objects, the stripes are placed hence at both sides of the 


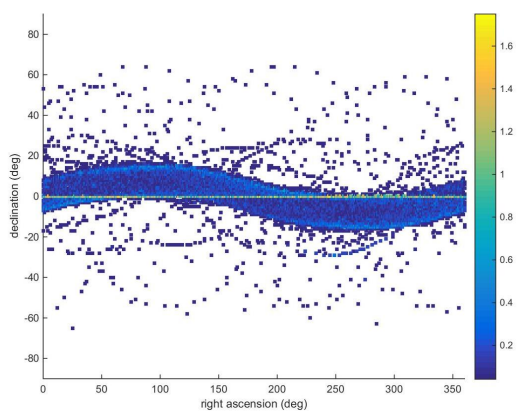

(a) $2 \mathrm{D}$ projection

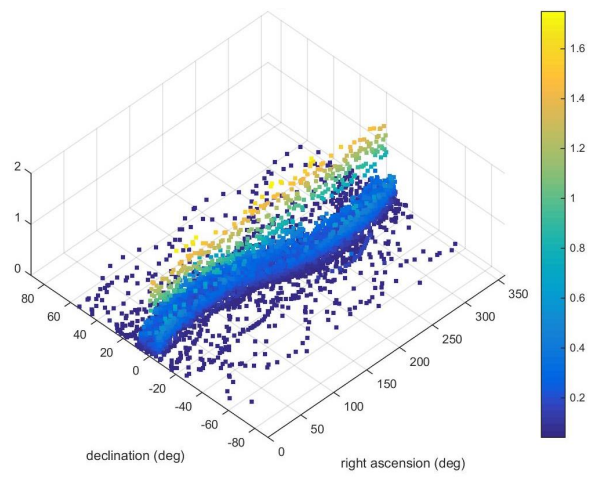

(b) $3 \mathrm{D}$ view

Fig. 2: Binned TLE catalog object positions over the night relative to the Zimmerwald topocentric location and chosen declination stripes.

Earth shadow; the first stripe is observed until the second stripe rises, then the two stripes are observed in parallel, until the first one sets. This setup expects to have an improved illumination and hence probability of detection and also allows for a larger anomaly spacing, however, not all objects that are detected are guaranteed to be observed twice even within the orbital planes that are covered theoretically by the observed declinations. The rise and set of the stripes leads to a number of objects that are only observed once.

\section{Orientation towards the Known Objects}

At which right ascensions the stripes are placed exactly and how many declination fields are chosen, is usually not decided using first principles, but is rather oriented at the objects currently listed in the US Strategic Command two-line element catalog (short: TLE catalog). Figure 2 shows the binned the location of the TLE catalog objects of July 12, 2016, averaged over 24 hours and the operationally chosen declination stripes for SMARTnet in July 2016; the earth's shadow is between the two stripes. The number of declinations are chosen such that the majority of the orbits are covered.

For uncontrolled geosynchronous objects, the lunisolar perturbations in combination with the Earth axis orientation relative to the ecliptic and Earth gravitational field lead to a perturbation of the orbital plane to plus minus 15 degrees in orbital inclination, that is the inclinations between zero and 15 degrees and larger than 345 degrees, respectively. The inclination change has a period of 53 years and is coupled with the progression of the right ascension of the ascending node. The operational satellites are kept at zero inclinations. It is already obvious that there is a trade-off between covering all inclinations of interest, which corresponds to the number of single declinations that are touched upon, which have a high potential to bear objects and to be leak proof. Furthermore, the number of single observation frames have to be sufficient to form a tracklet that rules out false detections. When two stripes are observed, the cycle time is generally increased because of the repositioning, when switching between the stripes.

\section{Surveys as Optimization Problem}

Instead of balancing these principles empirically, the problem can be viewed as an optimization problem in the sense of abstracting from the heuristic principles of the traditional surveys. The parameters are the following: The overall aim of all surveys is to detect the most objects possible and keep custody of them, that is to re-observe them adequately. In the initial detection, the maximum amount of the sky is sought to 
be observed. This can simply be obtained by spacing the observed viewing directions by the size of the field of view, such that no viewed areas overlap and to be re-observed before all possible viewing directions have been observed. However, the decision has to be made, what are best field of views to be observed. The following criteria need to be maximized:

1) initial detection

2) initial orbit determination of new objects

3) keeping custody/orbit improvement of known objects

The first principle is equivalent to maximizing the probability of detection in a twofold:

1.1) probability of detection when the object is in the field of view (illumination etc.)

1.2) the number of objects observed simultaneously in the field of view per viewing direction

The first item refers to getting the best possible signal to noise ratio (SNR) for the objects that are sought to be detected. The latter just means it is not very advantageous to observe regions of the sky, which contain no objects (if known beforehand).

The second principle entails that observations have been collected in a manner, that a first orbit determination without a priori information is possible. First orbit determination methods include classical solutions to Lambert's problem, or the time-ordered equivalent using Gauss or Lagrange method. The orbit improvement, the third principle, aims to collect observations, to update the probability density functions that are already known. Through the non-linear propagation in the presence of process noise, the uncertainty grows over time. To keep an object in custody observation updates are needed allowing the estimation an updated orbit with decreased uncertainty. The second and the third principle can be merged: Under the assumption of a first orbit determination based on a single tracklet with large initial uncertainties, such as in an admissible regions approach $[20,21,30]$.

\section{A. Formulation for the Maximum Observed Weighted Areas}

Formulating the problem in the standard form leads to a the constrained optimization problem:

$$
\begin{aligned}
\max A= & \sum_{g=1}^{l} \sum_{h=1}^{r_{g}} \sum_{f=1}^{m_{g}} \sum_{t_{g, f}=1}^{j_{g}}\left[\left(\sum_{i=1}^{n} \mu_{\mathrm{past}}\left(\alpha_{i}\left(t_{g, f}\right), \delta_{i}\left(t_{g, f}\right)\right) \cdot p\left(\alpha_{i}\left(t_{g, f}\right), \delta_{i}\left(t_{g, f}\right), \mathbf{o}, t_{g, f}\right) \cdot d\left(\alpha_{f, g}, \delta_{f, g}, \alpha_{i}, \delta_{i}, t_{g, f}\right)\right)\right. \\
\left.+k\left(\alpha_{f, g}, \delta_{f, g}, t_{g, f}\right)\right] & \\
\alpha_{f, g}-\frac{1}{2} \mathrm{FOV}-\alpha_{i}\left(t_{g, f}\right) & \leq 0 \\
-\alpha_{f, g}-\frac{1}{2} \mathrm{FOV}+\alpha_{i}\left(t_{g, f}\right) & \leq 0 \\
\delta_{f, g}-\frac{1}{2} \mathrm{FOV}-\delta_{i}\left(t_{g, f}\right) & \leq 0 \\
-\delta_{f, g}-\frac{1}{2} \mathrm{FOV}+\delta_{i}\left(t_{g, f}\right) & \leq 0 \\
\mathbf{R}-\sigma\left(\alpha_{i}, \dot{\alpha}_{i}, \delta_{i}, \dot{\delta}_{i}, \rho_{i}, \dot{\rho}_{i}, \nu\right) & \leq 0
\end{aligned}
$$


$A: \mathbb{R}^{k+4} \rightarrow \mathbb{R}$ being the cardinality of the weighted viewing direction areas, which is the quantity that is thought to be maximized. It is computed as the sum over all sensors $l$ that can be employed at any time in the optimization interval. The optimization interval can be for example a single night, a fraction thereof or cover an extended period, such as e.g. a month long observation campaign. The second sum is over the $r_{g}$ single time intervals for a given sensor $g$ within the optimization interval of length $t_{\mathrm{obs}, g, h}$. A sensor might not be available during the entire observation interval, for example optical sensors during the day (although this could also be handled via the probability of detection), because of maintenance downtime, or time-slots that are pre-allocated to other task in a shared use instrument. The third sum is over all $m_{g}$ viewing directions that are possible to be fit into the given observation interval for a given sensor. $m_{g}$ is determined as $m_{g}=\operatorname{int}\left(t_{\mathrm{obs}, g} / t_{\text {frame }, g}\right)$, with $t_{\text {frame }}=t_{\text {repos }}+j \cdot t_{\text {exp }}+(j-1) \cdot t_{\text {read }} \cdot t_{\text {frames }, g}$ is the time it takes the specific sensor to make a fixed number of frames $j_{g}$ with exposure time $t_{\text {exp }}$, readout time $t_{\text {read }}$ and repositioning time $t_{\text {repos }}$, for the last frame in a series, repositioning and readout can be done simultaneously. The optimization scheme is compartmentalized into the single time steps, $t_{f, g}$, that are corresponding to each of the $j_{g}$ exposures. The time discretization may be different for each sensor, based on the fact that observations are started as soon as the sensor is available in continuous time.

$\mu_{\text {past }} \in[0,1]$ is the orbit quality function. It is can be a deterministic or a probability function that is applied as a weighting function, depending on the individual objects. If the objects are sought to be observed disregarding any prior history, $\mu_{\text {past }}=1$. For all other cases, $\mu_{\text {past }}$ is a weight that rates, as of to when new observations are most benefiting to improve the orbital quality of the observed objects during the optimization interval. It is strictly periodic with the orbital period of the object. The orbit quality function is discussed in more detail in the next subsection.

$p\left(\alpha_{i}, \delta_{i}, \boldsymbol{o}, t_{g, f}\right) \in[0,1]$ is the probability of detection of the single object of interest located at right ascension $\alpha_{i, \mathrm{f}}$ and declination $\delta_{i, \mathrm{f}}$ at the time $t_{g, f}$. The probability of detection is not only dependent on the astrometric position of the objects, but also on other parameters, conflated under $\boldsymbol{o} \in \mathbb{R}^{k}$ including distance to the observer and the sun, location of the sun and object-dependent parameters, such as the object's size, shape, attitude, and surface reflection properties. In the chosen representation, the function $d\left(\alpha_{f, g}, \delta_{f, g}, \alpha_{i}, \delta_{i}, t_{g, f}\right): \mathbb{R}^{4} \rightarrow \mathbb{R}$ has been defined to represent the sensor to object association, for sensor $g$ and object $i$ at time $t_{g, f}$ for a given viewing direction of the sensor right ascension and declination, $\alpha_{f, g}, \delta_{f, g}$, respectively, at the same time. The association function is one, when the boundary conditions (Eq. 6 to 10) are fulfilled and zero otherwise. Theoretically one could merge functions $p$ and $d$ into one function, defining that the probability of detection is zero when the object is not in the field of view (FOV) of the sensor, however for cleanness of representation and ease of understanding the more extensive definition has been selected, treating probability of detection as a weighting function for the association.

The constraints serve a twofold purpose: For one, Eq. 6 to 9, the objects can only be observed if they are in the field of view (FOV) $(d=1)$ at the time of observation $t_{g, f}$ for given objects and viewing directions. Currently, the location of the object $\left(\alpha_{i, \mathrm{f}}, \delta_{i, \mathrm{f}}\right)$ is identified by the mean of its object probability density function (pdf) at that time. Secondly, Eq. 10 represents the re-observation constraint. The association is only valid $(d=1)$ when the object specific function $\sigma\left(\alpha_{i}, \dot{\alpha}_{i}, \delta_{i}, \dot{\delta}_{i}, \rho_{i}, \dot{\rho}_{i}, \nu, t_{g, f}\right): \mathbb{R}^{6+q} \rightarrow \mathbb{R}^{r}$ is above or at a threshold $\mathbf{R} \in \mathbb{R}^{r} . \sigma$ is a function of the full state of the object at minimum and potentially other parameters represented in $\nu$ or explicit time dependence. One interpretation of $\sigma$ could be the object pdf covariance. This way, objects are actively sought to be observed when they experience a covariance above a certain threshold, and do not count towards $A$, when their covariance is small. This leads to holding objects 
in custody, when for example $\mathbf{R}$ is set to be half of the field of view in the project right ascension, declination direction. Voluntarily, the two orbit criteria have been chosen, threshold $U$ and the orbit quality function $\mu_{\text {past }}$. Theoretically, the two could be combined to avoid a cutoff threshold $\mathbf{R}$, however this comes at the disadvantage of disregarding the dynamical distinctions of the problem. Hence, it has been chosen to clearly separate the effects that are periodic with the orbital period from the secular effects in the optimization.

In the absence of already observed objects and their location (pdfs respectively) or other a priori knowledge, the first half of Eq.5 is zero. It will automatically be populated as soon as objects have been observed and orbit determination has been performed. In order to be able to start a scenario without a prior information, or maintain a scenario not based upon single objects, the function $k\left(\alpha_{f, g}, \delta_{f, g}, t_{g, f}\right): \mathbb{R}^{k} \rightarrow \mathbb{R}$ has been introduced. $k$ can be understood as a multi-variate weighting function that is projected in the observation plane of right ascension and declination. The potentially continuous function leads to a scalar value for each viewing direction $\alpha_{f, g}, \delta_{f, g}$ for a given sensor at a given time $t_{g, f}$. The pdf itself can be hence understood as mapping out probability in the physical surveillance space. A representation of $k$ binned in right ascension and declination relative to an observer an be seen in Fig.2. Based on the TLE catalog, regions have assigned values between zero, no objects are likely to be present, to the maximum number of objects are present within the $24 \mathrm{~h}$ period. Alternatively, $k$ can be based upon regions of interest defined by a user or derived from astrodynamic principles from scratch for all objects or objects of interest. $k$ has the advantage that it does not have to define single objects that are counted but simple values for given viewing directions. $k$ can also be normalized to represent a probability measure.

When computing the optimization, either $k$ or $p \cdot d$ or both can have assigned values. The advantage of the formulation given in Eq.5 to 10 is that no hard decisions need to be encoded, but the optimization itself balances the single objects, initial detection upon a priori information or first principles and keeping custody of the objects that have been already observed.

The problem can be solved if it fulfills the convexity criteria both, in time, that is at every time step, and over time as the objects are not static. Although, the problem is solvable (at least for static objects), e.g. via branching methods, it is NP-hard, with object numbers of around $n \approx 1300$ (corresponding to the number of GEO objects currently in the TLE catalog) would take a long time to provide an exact solution. The pick of the viewing direction of the first frame even for a single sensor influences the viewing direction of all subsequent viewing directions. Solutions for the problem are along the lines of branch and bound algorithms, and sequential methods.

\section{B. Discussion of the Orbit Quality Weighting Function}

The orbit quality probability function is a periodic function. Based on past observations, not all observations along the orbit have the same impact on improving the obit quality. There are several ways to evaluate how the orbit quality can be best improved. One way is to use observability as a measure, as shown in $[7,8]$. The best point to re-observe the object is determined when the observability, evaluated via singular values of the observability matrix reaches its peak point in the smallest (most crucial) singular values. The observability is determined via the observability matrix:

$$
\mathcal{O}=\sum_{k=1}^{N} \boldsymbol{\Phi}\left(t_{k}, t_{0}\right)^{T} \mathbf{H}\left(t_{k}\right)^{T} \mathbf{H}\left(t_{k}\right) \boldsymbol{\Phi}\left(t_{k}, t_{0}\right),
$$



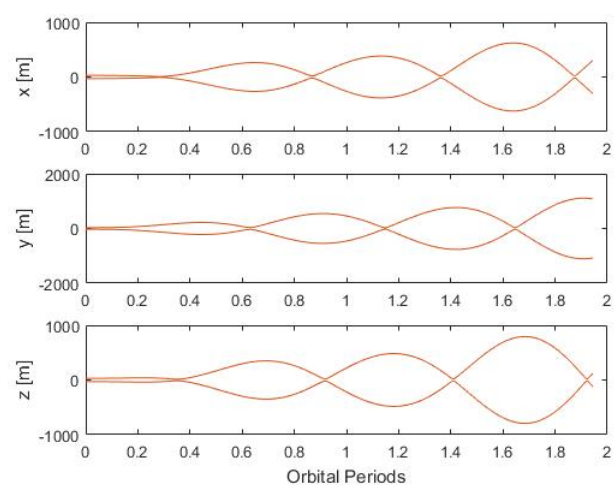

(a) Position $3 \sigma$
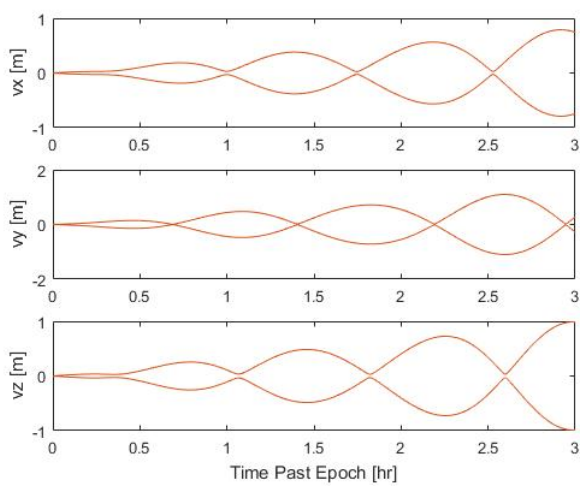

(b) Velocity $3 \sigma$

Fig. 3: Time history of the diagonal elements of the covariance matrix in a propagation only scenario without process noise. Plotted are the $3 \sigma$ (three times the standard deviation) boundaries.

where $\mathbf{H}$ is the linearized measurement matrix and Phi is the state transition matrix $\mathbf{\Phi} . N$ is the number of measurements that are taken into account. Alternatively, information measures have been evaluated [2, 3] based on the orbital probability density functions. In a propagation only scenario, even without process noise, the diagonal elements (position and velocity) in the covariance matrix $\mathbf{P}$ is growing over time, but because of the cyclic nature of the orbit problem, every component by itself undergoes periodic changes Fig.3. In order to evaluate the best time to re-observe the object, the Kullback-Leibler information gain has been utilized. It takes the following form under the assumption of a Kalman filter measurement update [3]:

$$
G_{\mathrm{KL}}=\frac{1}{2} \log \frac{\left|\mathbf{P}^{-}\right|}{\left|\mathbf{P}^{+}\right|}
$$

where $\left|\mathbf{P}^{-}\right|$denotes the determinant of the propagated covariance matrix prior to the update and $\left|\mathbf{P}^{+}\right|$the posterior updated covariance. It has to be noted that it does not have to be assumed that the prior and posterior mean is identical. It can clearly be seen in Fig.4a that the information gain spikes along the orbit, just prior to the half and a full orbit. As the overall covariance diagonal increases over time, the information measure also increases over multiple orbits (unlike the observability). For the computation, an actual update has to be computed. However, as shown in Fig.4b, the same trend can be observed without an actual update in evaluating only the innovation covariance $\mathbf{W}$. For zero measurement noise this is simply:

$$
\mathbf{W}=\mathbf{H P}^{-} \mathbf{H}^{T}
$$

Which is directly used to compute the Kalman gain in the update step. It can be understood as the projection of the propagated covariance in the measurement space. The observation time is best when the covariance in the measurement domain is the largest. As the latter is the simplest form, it can directly computed alongside the object propagation in the sensor tasking. Although both, Kullback-Leibler divergence and the innovation covariance approach are intuitive, they are not directly suitable for sensor tasking, as both quantities increase over time. This makes sense, as for example with the Kullback-Leibler divergence the more uncertain the object state becomes, the more information is gained updating with a precise measurements. However, this is undesired, because if the position uncertainty is much larger than the FOV of the sensor, the object will not be directly detected. The only workaround are then time-consuming sensor search patterns, to re-detect the object and obtain a measurement. One solution is the normalization of either quantity with respect to 


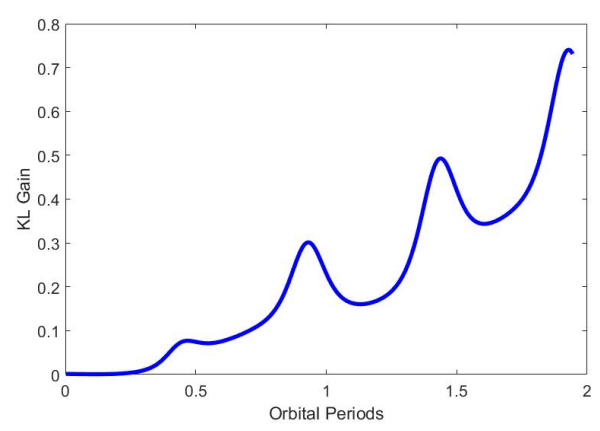

(a) KL information gain

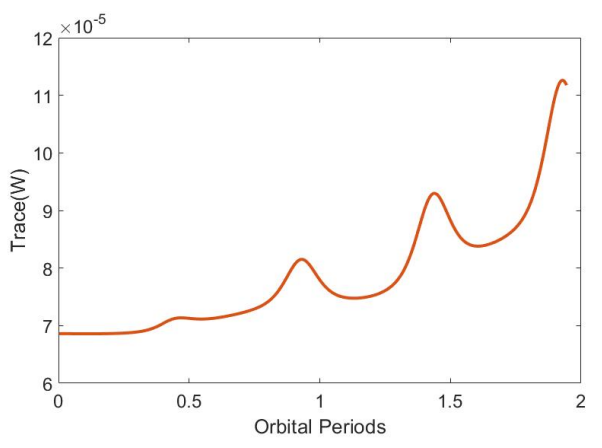

(b) Trace Innovation Covariance

Fig. 4: Time history of the Kullback-Leibler information gain and of the trace of the innovation covariance.

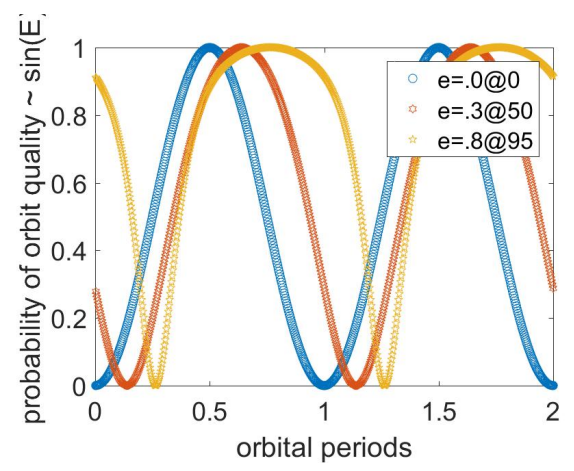

Fig. 5: Probability of orbit quality for three different orbits with first observation at true anomalies of zero, 50 , and 95 degrees and eccentricities of zero, 0.3 , and 0.8 .

one orbital period. For an even simpler implementation, for example when orbital uncertainties are unknown and only means are propagated, a simplified sinusoidal curve can be chosen of the eccentric anomaly $E$, to mimic the known uncertainty pattern in the measurement space. It is shown in Fig.19.

\section{Near-Optimal Solution of the Optimization Problem}

To reduce the computational burden, the problem is reformulated, allowing for a near-optimal solution of the problem.

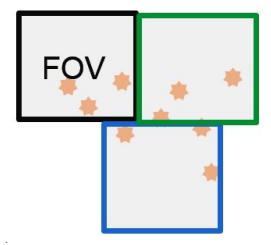

(a) Tasking option 1

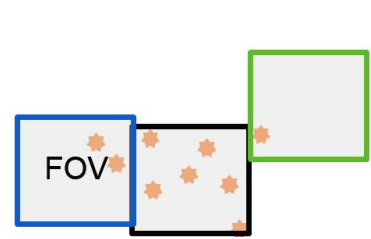

(b) Tasking option 2

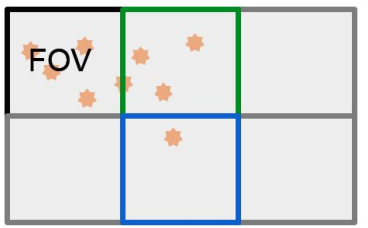

(c) Tasking on grid points

Fig. 6: Tasking scenarios: a) and b) show two very similar but still distinct tasking options of viewing directions to cover the symbolic targets in yellow. c) is showing tasking along fixed grid points. 


\section{A. Average Time Grid Formulation}

Fig.6a) and b) illustrates that very similar selections of viewing directions of the sensor to cover a fixed number of targets; leading to a very similar performance at a tremendous computational cost. Instead, fixed viewing directions are defined for allowed viewing directions. That is only fixed grid points in the right ascension and declination space can be visited, Fig.6c). The grid points are chosen based on the FOV of the sensors that are used, to not have gaps in the sky coverage. This means grid points can differ for each sensor.

The second approximation that is made to avoid one of the sums of expression Eq. 5. Instead of optimizing for the exact observation times $t_{g, f}$, which are $j_{g}$ time steps for each viewing direction, the mid-exposure time of the series is selected as the optimization criteria. This would be one time $m_{g} \rightarrow \tau_{g, f}$ for each observation direction $f$. The downside is that the proposed strategy is only optimal for the given grid for the mid-series time and not in general.

\section{B. Global and Local Optimum}

A local optimum of the problem can easily be found in selecting the area with the highest weight at each time step. The local optimum, however, does not coincide with the global optimum, in general. This is the only the case when no conflicts exist. Conflicts can be divided into two groups, in-time conflicts, and over-time conflicts. In-time conflicts mean that for the same time step more than one viewing area has the same value. It is hence not immediately clear, which one to choose. Over-time conflicts are, however, more severe. This means that at a time a viewing direction area might have a lower value, but at later times, selecting the lower value area will lead to the optimal overall result. This can happen in our case when an object is not visible at later times and hence has to be observed earlier. A second example would be the orbit quality, that might be improved for all objects, when not selecting the highest rated area at each time step independently. The latter, however, is already taken care of, via the orbit quality weighting of the areas. For the prior, a new function is introduced, the so-called urgency function. The urgency function $u(t)$ is simply evaluating for how long an object is visible in the given optimization scenario and creating a function that linearly increases with the decrease of the remaining time at any given time step. Thereby it does not matter if there are times in between during which the object is not visible (for example when going through earth shadow). The function simply increases the value of the specific viewing direction of objects which are visible shorter times relative to those that are visible at longer times. In an optimal solution to the problem, the urgency function is superfluous and potentially worsens the overall result. In the local optimal evaluation, it uses the known dynamics of the objects to reduce over-time conflicts. As a side effect, it also diversifies the values at the same time step and hence reduces in-time conflicts as well. A visualization for the GEO objects in the TLE catalog at the given scenario of one observation night is given in Fig.7.

\section{Near-Optimal Formulation}

This leads in summary to the following, altered, computationally faster formulation of the optimization problem, described in Eq.5 that is suited for evaluation via the local optimum:

$$
\begin{gathered}
\max \tilde{A}=\begin{array}{c}
l \\
\sum_{g=1}^{l} \sum_{h=1}^{r_{g}} \sum_{f=1}^{m_{g}}\left[\left(\sum_{i=1}^{n} u\left(\tau_{g, f}\right) \cdot \mu_{\text {past }}\left(\alpha_{i}\left(\tau_{g, f}\right), \delta_{i}\left(\tau_{g, f}\right)\right) \cdot p\left(\alpha_{i}\left(\tau_{g, f}\right), \delta_{i}\left(\tau_{g, f}\right), \mathbf{o}, \tau_{g, f}\right) \cdot d\left(\alpha_{f, g}, \delta_{f, g}, \alpha_{i}, \delta_{i}, \tau_{g, f}\right)\right)\right. \\
\left.+k\left(\alpha_{f, g}, \delta_{f, g}, \tau_{g, f}\right)\right]
\end{array}
\end{gathered}
$$




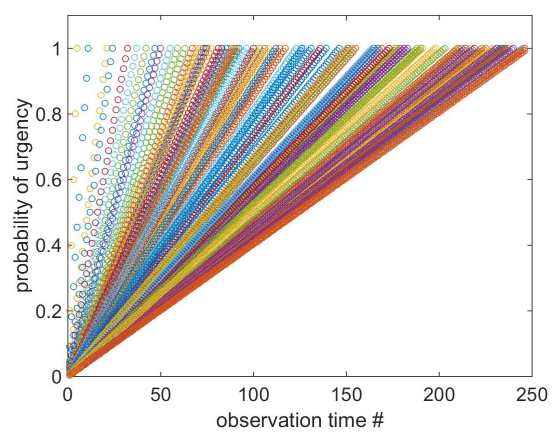

Fig. 7: Diversification in time and over time for local optimization via the urgency probability function.

$$
\begin{aligned}
\alpha_{f, g}=\left\{\alpha_{1}, \alpha_{2}, \ldots, \alpha_{m}\right\}, m & \in \mathbb{N} \\
\delta_{f, g}=\left\{\delta_{1}, \delta_{2}, \ldots, \delta_{m}\right\}, m & \in \mathbb{N} \\
\alpha_{f, g}-\frac{1}{2} \mathrm{FOV}-\alpha_{i}\left(\tau_{g, f}\right) & \leq 0 \\
-\alpha_{f, g}-\frac{1}{2} \mathrm{FOV}+\alpha_{i}\left(\tau_{g, f}\right) & \leq 0 \\
\delta_{f, g}-\frac{1}{2} \mathrm{FOV}-\delta_{i}\left(\tau_{g, f}\right) & \leq 0 \\
-\delta_{f, g}-\frac{1}{2} \mathrm{FOV}+\delta_{i}\left(\tau_{g, f}\right) & \leq 0 \\
\mathbf{R}-\sigma\left(\alpha_{i}, \dot{\alpha}_{i}, \delta_{i}, \dot{\delta}_{i}, \rho_{i}, \dot{\rho}_{i}, \nu\right) & \leq 0
\end{aligned}
$$

Which has in comparison to the formulation Eq.5 the two new constraints, concerning the grid points, Eq.17 and 18. In the formulation Eq.14 the urgency function has been added. Therefore, the sum over the single exposure times has been replaced with the mid-series time $\tau_{g, f}$. Again this problem can be solved via integer math formulations of the association problem. One has to keep in mind that optimality of the full problem cannot be guaranteed any more because only the approximated problem is solved for now. This also means $\max \tilde{A} \leq \max A$. In most of the cases, the approximate problem will perform worse than the exact problem $\max \tilde{A}<\max A$. In order to speed up computation time, using a greedy algorithm, that is choosing the local optimal allows for a rapid computation of sensor tasking scenario. As a result no iterations are needed. The near-optimal scenario is very fast to compute even on a regular single core machine. The dominating time factor is not the optimization but the propagation time of all the objects. Real-time applications and online updating or adaption of the strategy are hence possible without extended computational facilities for SGP4 or other three degree of freedom standard propagators.

\section{Earth Shadow}

For the Earth shadow computation, a very simplistic cylindrical model has been adapted. The object is in Earth shadow, with no illumination if:

$$
\varangle_{\text {sun,sat }}>\pi-\arcsin \frac{R_{\text {earth }}}{\left|\mathbf{x}_{\text {sat }}\right|}
$$

where $\varangle_{\text {sun,sat }}$ is the angle between the geocentric sun and the geocentric object vector, $R_{\text {earth }}$ is the mean radius of the Earth, and $\left|\mathbf{x}_{\text {sat }}\right|$ is the momentary distance of the satellite located at $\mathbf{x}_{\mathrm{sat}}$. For more general use, 
the distance to the satellite can also be exchanged with the general geosynchronous distance of 42'000km. Although it is well known, that especially in the geostationary region earth shadow is usually not complete especially in the red spectrum, and that there is a smooth transition in the illumination condition, this simplified scenario has been used to see the most harm to the object coverage that can be done by an Earth shadow that forces the probability of detection down to zero immediately, independent of object sizes etc.

\section{Simulation Results}

Simulations have been made for according to two SMARTnet sensors two different sensors have been modeled: One holding a 3.77 x 3.77 degrees FOV, denoted large FOV, or LFOV in the following, and one sensor holding a 0.6115 by 0.6115 degrees FOV denoted small FOV, or SFOV, respectively. Both sensors are assumed to be located in Zimmerwald, Switzerland. The sensor characteristics besides the field of view are chosen to be identical, simulating two different cameras on the same mount, utilizing the same optics. Oriented towards the real values, an exposure time of eight seconds, readout time of seven seconds. For the classical stripe scanning strategy, it is assumed that repositioning within the stripe takes nine seconds and a repositioning to the beginning or the next stripe 30 seconds. The rationale behind this that it might take less time in the command structure of the telescope is set to the stripe scanning or has specific means to suppress vibrations during the inner-stripe repositioning. For the new optimized strategy, the larger repositioning value of 30 seconds is assumed. This presumes a standard telescope mount setup, where the repositioning time is not driven by the speed of the telescope mount and hence by the distance by which the telescope is moved, but by the settling time of the telescope, to be able to take a stable image. Seven exposures per declination point are taken in both strategies. Several exposures per viewing direction are necessary to confirm detections in the presence of cosmic ray contamination and for objects, which are either very faint in general or at times. From the exposures, a so-called tracklet is formed. Using the tracklet, a first restricted orbit determination or an admissible region, mapping out the possible range and range rate space, can be formed.

In the following two different scenarios are exactly simulated, using the USSTRATCOM two-line element (TLE) catalog. In the first scenario, the aim is to have one observation per visible object. In the second scenario, two observations per detected object are sought. This most closely resembles a scenario without any a priori information, for which at least two tracklets per newly detected object are needed, in order to have a good enough orbit to successfully find the object again in the next night. Each of the scenarios are during the July 122016 summer night, within sunset to sunrise, comprising an observation time of 361 minutes. In all scenarios, probability of detection is one during the observation time span, except when the objects are below local horizon or in Earth shadow. The classical stripe scanning scenario the stripes are placed outside the Earth shadow, naturally. A total of 1285 objects, all the geosynchronous objects in the catalog at July 12, have been used; 506 objects are visible for the observer location within the observation time span. The visible objects are understood as any object that was visible during any point in time during the night. They are plotted in Fig. 8 with their positions at the end of the night. The limitations of the local horizon is clearly visible. It also shows that some high inclination objects, also must have high eccentricities, exposing a different relative velocity. Which means all visible objects are, theoretically at least, observable. Not all objects, that are counted as visible, are visible during the whole night. For the optimized scenario, the approximation in Eq. 14 has been used, evaluating at the local optimum. In the optimization computation the main computationally limiting factor is the object propagation to the mid-observation times even in a brute force implementation as the only other computation per object that is required is the analytic computation of 
the weighting and which grid field the object is currently in.

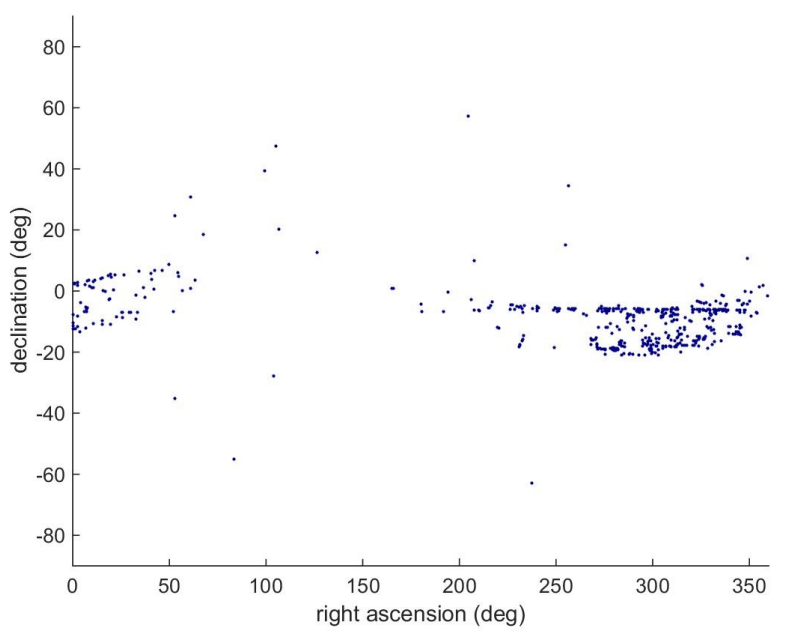

Fig. 8: All near geosynchronous objects that are visible in at July 12, 2016, plotted at their position at the end of the observation scenario.

A. One Observation per Geosynchronous Mean Motion Object, Probability of Detection One

1. Classical One Stripe Scanning

In the classical scenario, when one observation is sought, a one stripe scanning strategy can be employed. Fig.14 shows the placement of the stripe on top of the averaged TLE objects. The figure shows the placement of two stripes. For the one stripe strategy, the right stripe is selected. The placement is close the Earth shadow, to ensure maximum visibility of the objects. To cover the whole declination of likely objects to pass through, six declination stripes are used for the LFOV setup. The small FOV setup would normally not be used for surveying, for the sake of illustration, we adopt the same strategy, which leads to defining 29 declinations, in order to cover the same declinations. Fig9 shows the detected objects versus the undetected ones after completion of the night. Because the SFOV setup is not leak-proof, a lot of objects, which are theoretically passing the stripe are not caught in actual observations. Fig.10 shows the rate of the detected objects out of the visible ones. The rate of detected objects is about 70 percent for the LFOV, a telescope that would usually be applied for the surveys, and only about 6 percent for the SFOV.

\section{One Observation per Object Optimized Strategy}

The new near-optimal strategy is scanning the same net area of the sky during the night. As a solution, the local optimization under the use of the urgency function has been used. Fig.11 shows the chosen areas, which the telescope visited, using the SFOV and the LFOV setup, respectively. The colors indicate the weight of the chosen area. It has to be noted that the telescope had idle time as it had covered all visible objects. This time can be used to scan addition areas, via populating the function $k$ in Eq.5, for example. Fig.12 shows the detected and undetected objects for both configurations at the end of the night. Keep in mind that not all objects are above the local horizon during the night; this is especially true for the objects at right ascensions between 20 and 200 for the chosen observation station position. Fig.13 illustrates the rates of observed objects versus all visible ones. The rate of the detected objects is 100 percent of the visible ones for 


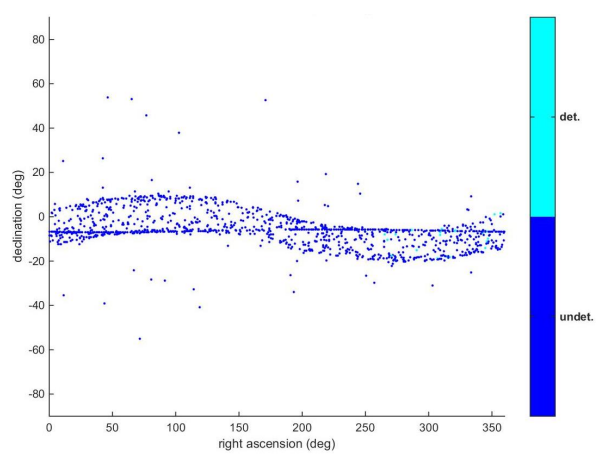

(a) SFOV

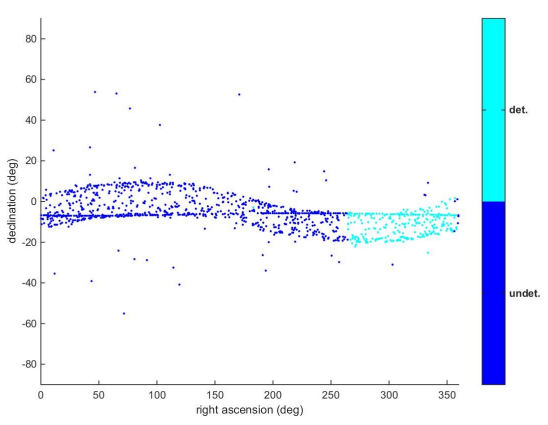

(b) LFOV

Fig. 9: Simulation of the detected and undetected objects at the end of the observation night Zimmerwald observer with a one-stripe strategy for a small field of view (SFOV) and a large field of view (LFOV) setup.

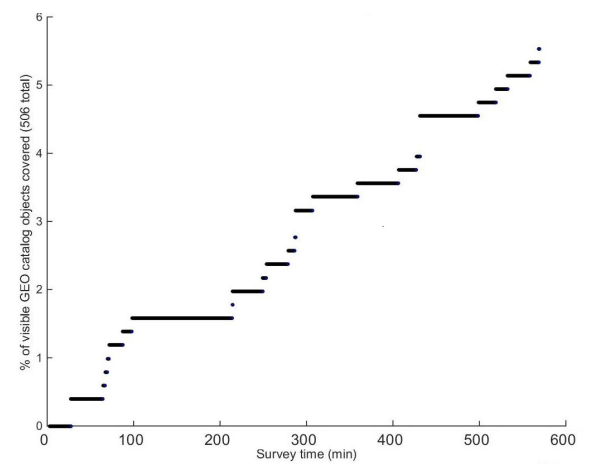

(a) SFOV

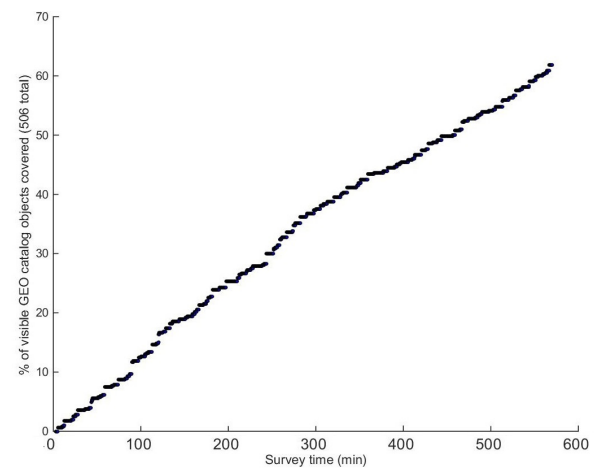

(b) LFOV

Fig. 10: Rate of detected objects for a Zimmerwald observer on July 12, 2016 with a one-stripe strategy for a small field of view (SFOV) and a large field of view (LFOV) setup.

the LFOV setup. For the SFOV, the coverage is necessarily less, although even with the significantly smaller FOV, about 73 percent of the objects can still be observed. This means, even with the SFOV, successful observation campaigns can be made with the correct strategy.

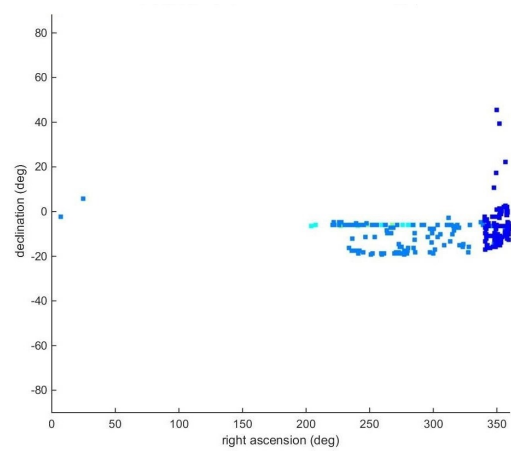

(a) SFOV

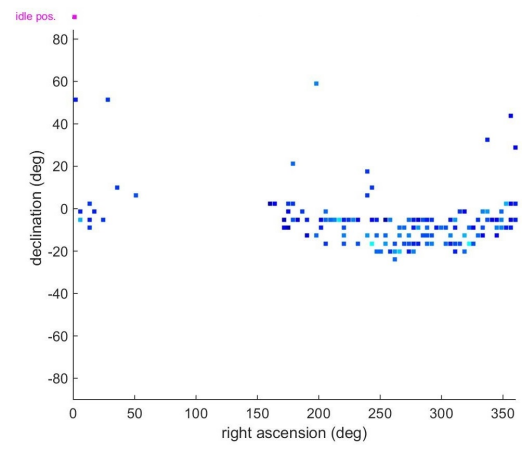

(b) LFOV

Fig. 11: Chosen grid point viewing directions (b) SFOV and (c) LFOV by the optimization scheme for one observation per object. Lighter colors indicated higher field values.

When comparing to the optimized scenario even though a sub-optimal solution is brought forth, it out- 


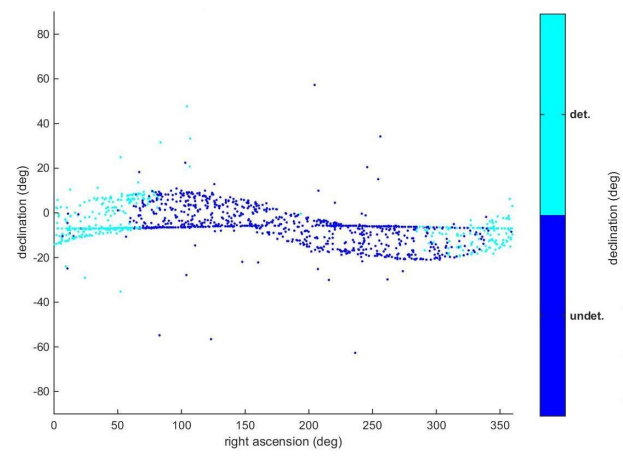

(a) SFOV

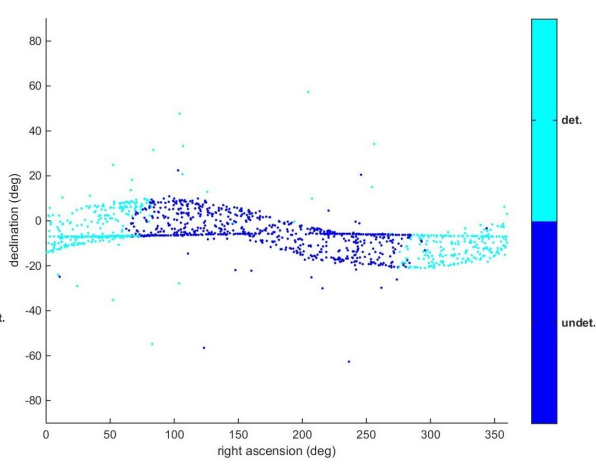

(b) LFOV

Fig. 12: July 12, 2016 detected (turquoise) and undetected objects (dark blue) with the optimization scheme for one observation per object for a Zimmerwald observer.

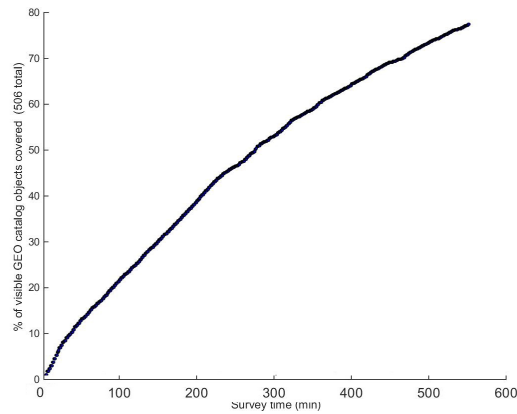

(a) SFOV

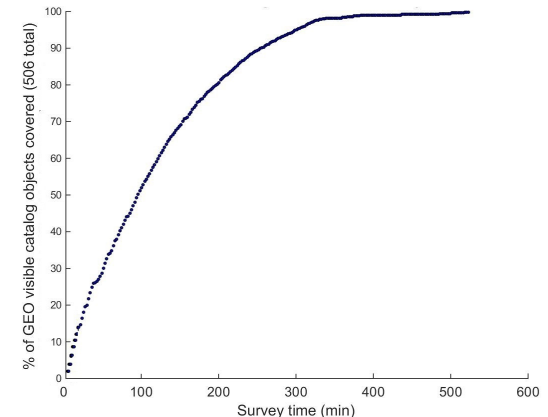

(b) LFOV

Fig. 13: July 12, 2016 detected object rate the optimization scheme for one observation per object for a Zimmerwald observer.

performs the single stripe traditional survey strategy. This is obvious for the LFOV, where all visible objects are observed, but the most impressive results are comparing the observation rate of below ten percent in the traditional one stripe scanning and over 70 percent with the optimized strategy.

\section{B. Two Observations per Geosynchronous Mean Motion Object, Probability of Detection One}

\section{Classical Two Stripe Scanning}

In a more realistic scenario, two observations per newly detected object are sought within the same night, in order to allow for an initial orbit determination. In the classical scenario, this corresponds to a two stripe scenario. For SMARTnet, two stripes in the vicinity of the earth's shadow were planned, see Fig.14. When two stripes are observed in parallel with the given constraints on repositioning, the scenario is not leak proof any more even for the LFOV configuration. That is objects with a geosynchronous mean motion are passing by without being detected. This is clearly visible in Fig.15. This leads to a rate of just below 40 percent of all visible objects, see Fig.16. For the SFOV the situation is detrimental, because none of the objects is detected twice although posing a two-stripe strategy, see Figs.15 and 16. 


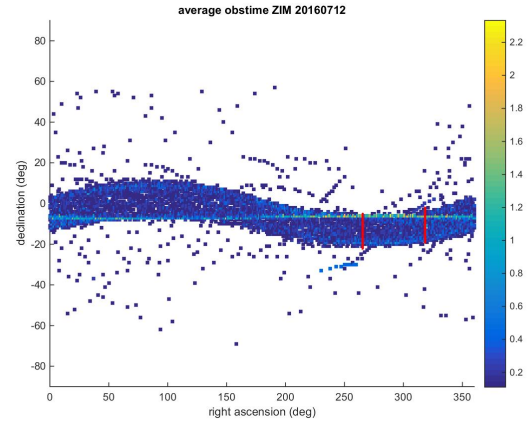

(a) LFOV

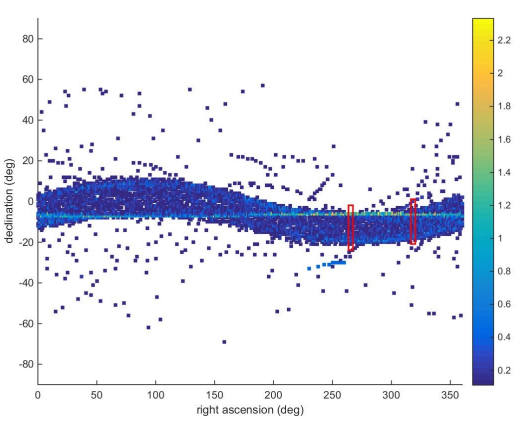

(b) LFOV

Fig. 14: Binned TLE catalog object positions over the night relative to the Zimmerwald topocentric location and chosen declination stripes. Both stripes for two stripe strategy, right stripe for one stripe strategy.

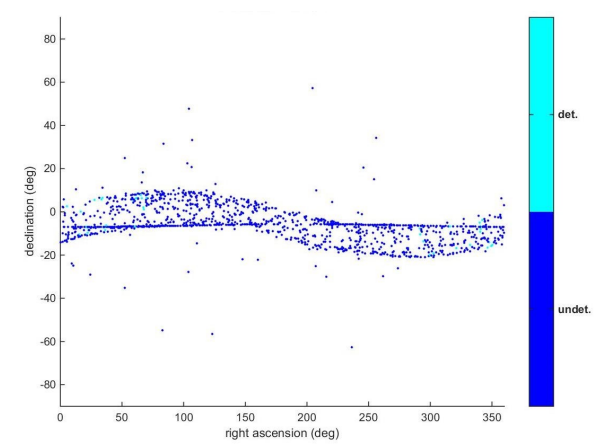

(a) SFOV

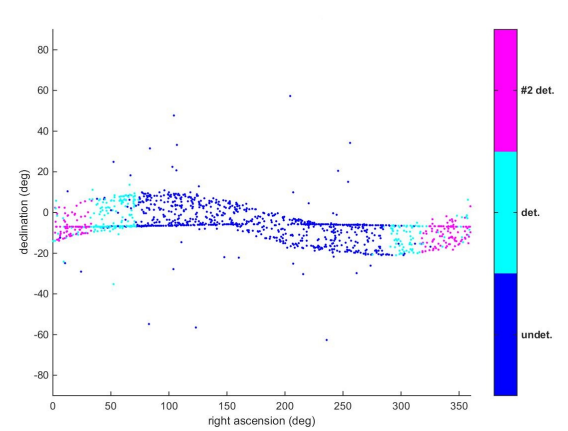

(b) LFOV

Fig. 15: Simulation of the detected (turquois) and undetected (blue) objects at the end of the observation night Zimmerwald observer with a two-stripe strategy for a small field of view (SFOV) and a large field of view (LFOV) setup.

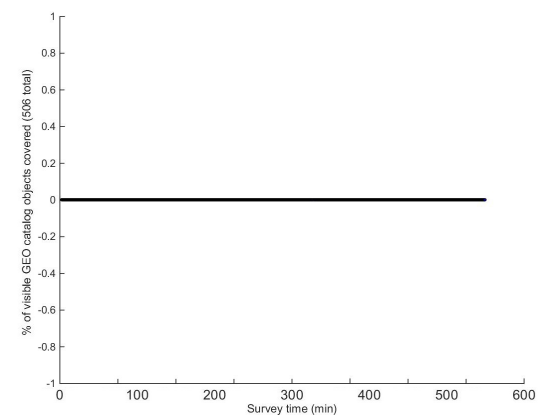

(a) SFOV

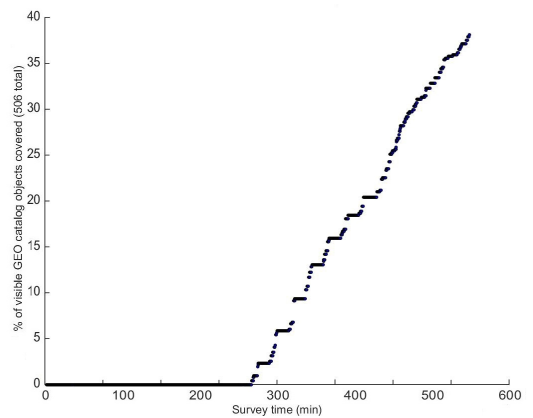

(b) LFOV

Fig. 16: Rate of detected objects for a Zimmerwald observer on July 12, 2016 with a two-stripe strategy for a small field of view (SFOV) and a large field of view (LFOV) setup.

\section{Two Observations per Object Optimized Strategy}

Fig. 17 shows the chosen grid point areas, which the telescope visited. In this scenario, no idle time is present for either of the configurations. Two observations with an advantageous anomaly difference are 
sought. In the LFOV, not all possible objects are visited, as it is already obvious from Fig.17, which shows the undetected, single detected and twice detected objects. Fig.18 reveals that still 80 percent of the visible objects are detected twice during the observation night. Initially, more new detections are made, towards the end of the night, more second detections are performed. Fig19 illustrates, that the observations are spaced out sufficiently in anomaly to be useful for a first orbit determination using two tracklets. In comparison, Figs.17,18, and 19 show the same scenario for the SFOV. Naturally, the detection rates are lower, but still, around 42 percent of the visible objects are in fact observed twice within a single night with comparable anomaly differences. Fig. 19 shows the anomaly spacing; most objects have anomaly difference of around 50 degrees or more. In all scenarios, it has to be noted that the inclusion of the exact Earth shadow, compared

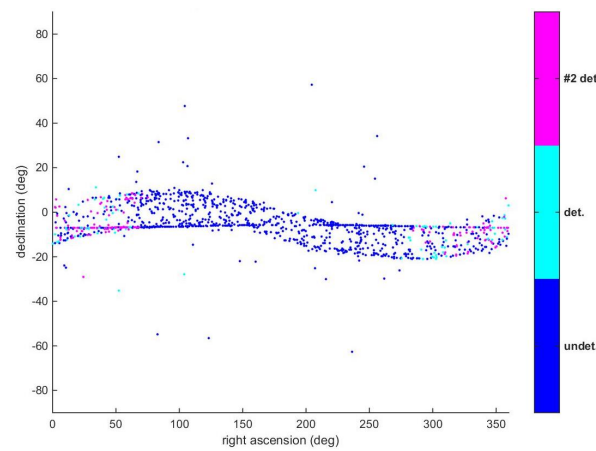

(a) SFOV

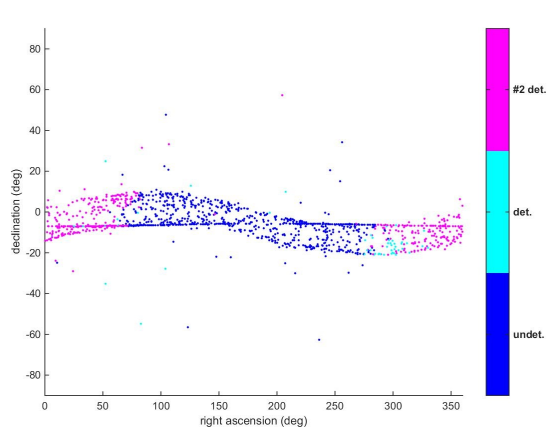

(b) LFOV

Fig. 17: July 12, 2016; objects detected once (turquoise), twice (magenta) and undetected objects (dark blue) for two observations per object for a Zimmerwald observer.

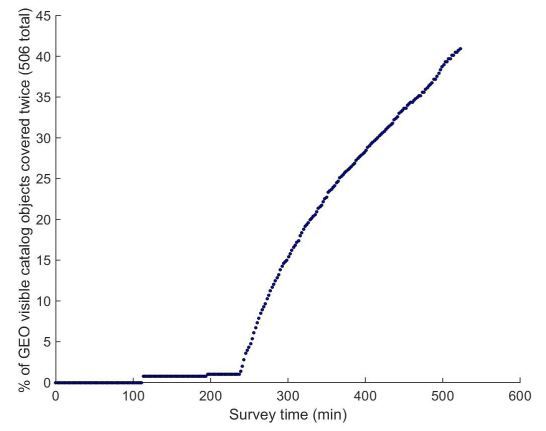

(a) SFOV

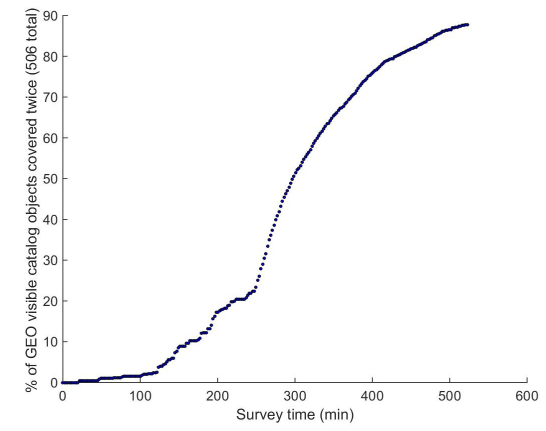

(b) LFOV

Fig. 18: July 12, 2016, detected object rate the optimization scheme for two observations per object for a Zimmerwald observer.

to runs excluding earth shadow does not lead to significant changes in the rates, the maximum difference was one object that was detected less with the SFOV. However, the sequence at which viewing directions were chosen is altered, depending on the Earth shadow inclusion. When comparing the optimized scenario with the traditional two stripe scanning, it can be observed that the optimized scenario outperforms the traditional two stripe survey. While in the optimized scenario even the SFOV leads to usable results of over 40 percent of the objects have been observed twice with a significant anomaly offset, re-observation with the two stripe scenario was not successful with the SFOV as zero objects have been re-observed. This is because the two stripes were not leak proof. Even with the large FOV, more than twice as many objects have been re-observed 


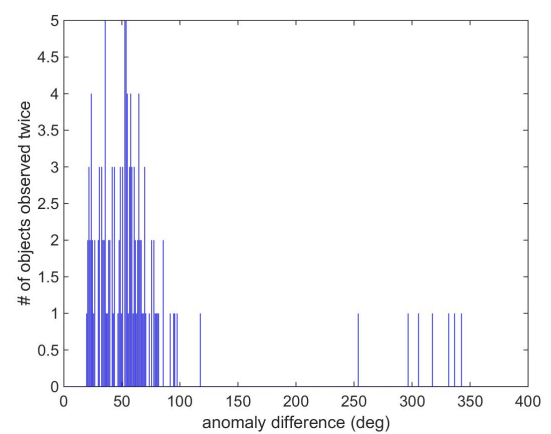

(a) SFOV

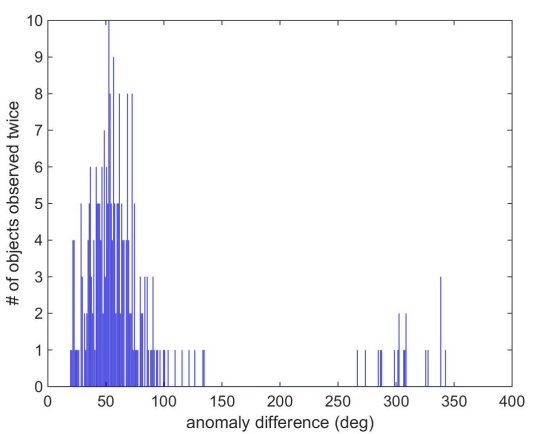

(b) LFOV

Fig. 19: July 12, anomaly differences between the two observation batches in the two observations per object optimized scheme.

with the optimized scenario than in the classical technique. Of course, it has to be taken into account that the classical techniques are blind techniques, not using the exact orbital elements of the objects. 


\section{Conclusions}

This paper investigated observation strategies for optical sensors. As an example, ground-based observations of the geosynchronous region have been used. Classical declination stripe observation strategies have been illustrated. They have been compared with a new observation strategy. In order to do so, the problem has been reformulated as an optimization problem translating heuristic principles into a rigorous framework. In order to reduce the computational complexity, a grid-based viewing direction formulation has been introduced. The portion of the sky that is viewed is optimized in weighing the different viewing directions by how many objects, that are either in the TLE catalog or via definition of regions with high probabilities for new objects, their visibility, and probability of detection. A user-defined threshold can be set about the desired orbital uncertainty before being scheduled for re-observation. Effectively that defines the desired number of detections of new objects within the optimization schedule, such as to observe each object only once or for example twice within one night.

A near optimal solution very fast to compute method is shown in further diversifying the weights both at the observation times and over the whole night, via the urgency and the anomaly probability formulations. The near-optimal solution can be obtained in real-time. Updates of the observation strategy online during the night are hence possible.

For performance illustration, two ground-based observation setups have been used, a probability of detection of one is assumed for all observations. A small field of view (SFOV) setup and a large field of view (LFOV) with a field of view of 0.6115 by 0.6115 and 3.7 by 3.7 degrees, respectively. The probability of detection has been set to one above local horizon and outside Earth shadow. With the traditional stripe scanning strategy, the LFOV observes about 63 percent of the visible objects once in a one stripe scanning strategy and around 40 percent twice in a two stripe strategy in a single summer night. Because the stripe is not leak-proof, the SFOV only observes around 6 percent successfully in the one stripe strategy and none twice in the two stripe strategy. In comparison with the optimized strategy, 100 percent including idle time are observed once with the LFOV setup, and when two observations per night are sought, over 80 percent of the visible objects are observed twice. For the SFOV the performance is also improved leading to 73 and 42 percent respectively. This shows that even with the small field of view, highly efficient observation campaigns with many objects are possible.

\section{Acknowledgments}

The author would like to acknowledge the support of this work by the SSA Group of GSOC, DLR, Oberpfaffenhofen, and thank Dr. Oliver Montenbruck for fruitful and tough discussions, Dr. Kyle DeMars for very helpful insights concerning information gain methods, and Martin Weigel for many useful comments on the manuscript.

\section{References}

[1] J. Africano, T. Schildknecht, M. Matney, P. Kervin, E. Stansbery, and W. Flury. A Geosynchronous Orbit Search Strategy. Space Debris, 2:357-369, 2000. doi: 10.1023/B:SDEB.0000030025.04930.08.

[2] E. Cordelli, A. Vananti, and T. Schildknecht. Covariance study to evaluate the influence of optical follow-up strategies on estimated orbital parameters. Acta Astronautica, 122:76 - 89, 2016. ISSN 0094-5765. doi: http: //doi.org/10.1016/j.actaastro.2016.01.020. 
[3] Kyle J. DeMars and Moriba K. Jah. Evaluation of the information content of observations with application to sensor management for orbit determination. volume 142 of Advances in the Astronautical Sciences, pages 3169 3188, 2011.

[4] J. Ender, L. Leushacke, and A. Brenner. Radar techniques for space situational awareness. In IEEE Radar Symposium (IRS), 2011 Proceedings International, 2011.

[5] J. Ferreira, I. Hussein, J. Gerber, and R. Sivilli. Optimal SSN Tasking to Enhance Real-time Space Situational Awareness. In Proceedings of the AMOS Technical Conference, 20-23 Sep, Maui, Hawaii, USA, 2016.

[6] T. Flohrer, T. Schildknecht, R. and E. Stöveken. Performance Estimation for GEO Space Surveillance. Advances in Space Research, 35(7):1226-1235. doi: https://doi.org/10.1016/j.asr.2005.03.101.

[7] A. Friedman and C. Frueh. Observability analysis applied to artificial near-earth objects with noise. Space Flight Mechanics Meeting, San Antonio, TX, February 2016. 27th AAS/AIAA Space Flight Mechanics Meeting. AAS 17-237.

[8] A. Friedman and C. Frueh. Determining Debris Characteristics From Observability Analysis of Artificial NearEarth Objects. In Seventh European Conference on Space Debris, ESOC, Darmstadt, Germany, 18-21 April, 2017.

[9] C. Frueh. Realistic Sensor Tasking Strategies. In Advanced Maui Optical and Space Surveillance Technologies Conference (AMOS), September 2016.

[10] C. Frueh. Sensor Tasking for Multi-Sensor Space Object Surveillance. In Seventh European Conference on Space Debris, ESOC, Darmstadt, Germany, 18-21 April, 2017.

[11] C. Frueh, S.N. Paul, and H. Fiedler. Sensor Tasking for Detection and Custody of HAMR Objects. In Advanced Maui Optical and Space Surveillance Technologies Conference (AMOS), September 2017.

[12] C. Früh and T. Schildknecht. Object Iimage Linking of Earth Orbiting Objects in the Presence of Cosmics. Advances in Space Research, 49:594 - 602. doi: https://doi.org/10.1016/j.asr.2011.10.021.

[13] S. Gehly and J. Bennet. A Sensor Tasking Reward Function Incorporating Target Priorities. In Advanced Maui Optical and Space Surveillance Technologies Conference, September 2016.

[14] S. Gehly, B. Jones, and P. Axelrad. Sensor allocation for tracking geosynchronous space objects. Journal of Guidance, Control and Dynamics, page https://doi.org/10.2514/1.G000421, 2017.

[15] J. Herzog, C. Früh, and T. Schildknecht. Build-up and Maintenance of a Catalogue of GEO Objects with Zimsmart and Zimsmart2. In Proceedings of the International Astronautical Congress 2010, A6.5.2, Prague, Czech Republic, 27 Sep-1 Oct, 2010, 2010.

[16] A. Jaunzemis, M. Holzinger, and M. Jah. Evidence-based Sensor Tasking for Space Domain Awareness. In Advanced Maui Optical and Space Surveillance Technologies Conference, September 2016.

[17] R. Linares and R. Furfaro. Dynamic Sensor Tasking for Space Situational Awareness via Reinforcement Learning. In Advanced Maui Optical and Space Surveillance Technologies Conference, September 2016.

[18] R. Linares and R. Furfaro. An Autonomous Sensor Tasking Approach for Large Scale Space Object Cataloging. In Advanced Maui Optical and Space Surveillance Technologies Conference, September 2017.

[19] B. Little and C. Frueh. Comparison of Optimizers for Ground Based and Space Based Survey Sensors. In AAS/AIAA Astrodynamic Specialist Conference, Stevenson, WA, 2017. 17-769.

[20] A. Milani, M.E. Sansaturio, and S.R. Chesley. The Asteroid Identification Problem IV: Attributions. Icarus, 151: 150 - 159, 2001. doi: https://doi.org/10.1006/icar.2001.6594.

[21] A. Milani, G.F. Gronchi, D. Farnocchia, G. Tommei, and L. Dimare. Optimization of Space Surveillance Resources by Innovative Preliminary Orbit Methods. In Proceedings of the Fifth European Conference on Space Debris, ESOC, Darmstadt, Germany, 30 March-2 April 2009, 2009.

[22] T. Murphy and M. Holzinger. Generalized Minimum-Time Follow-up Approaches Applied to Tasking ElectroOptical Sensor Tasking. In Advanced Maui Optical and Space Surveillance Technologies Conference, September 2017.

[23] R. Musci, T. Schildknecht, T. Flohrer, and G. Beutler. Concept for a Catalogue of Space Debris in GEO. In Proceedings of the Fourth European Conference on Space Debris, pp. 601-606, ESOC, Darmstadt, Germany, 1820 April 2005, 2005.

[24] R. Musci, T. Schildknecht, M. Ploner, and G. Beutler. Orbit Improvement for GTO Objects Using Follow-up Obervations. Advances in Space Research, 35(7):1236-1242, 2005. doi: 10.1016/j.asr.2005.02.074.

[25] A. Nafi and K. Fujimoto. A unified approach for optical survey strategy design of resident space objects. In 
AAS/AIAA Astrodynamics Specialist Conference, Sep 2016.

[26] J. Pelton. Tracking of Orbital Debris and Avoidance of Satellite Collisions, Handbook of Satellite Applications. 2016. Springer, New York.

[27] T. Schildknecht. The Search for Space Debris Objects in High-Altitude Orbits. Astronomical Institute, University of Bern, 2003. Habilitation treatise.

[28] T. Schildknecht, U. Hugentobler, and M. Ploner. Optical Surveys of Space Debris in GEO. Advances in Space Research, 62(1):45 - 54, 1999. doi: https://doi.org/10.1016/S0273-1177(98)00229-4.

[29] T. Schildknecht, W. Flury, C. Früh, J. Herzog, A. Hinze, and A. Vananti. Using Optical Observations to Survey, Track, and Characterize Small-Size Objects at High Altitudes. In Proceedings of 28th International Symposium on Space Technology and Science, June 5-12, Okinawa, Japan, 2011, 2011.

[30] G. Tommei, A. Milani, D. Farnocchia, and A. Rossi. Correlation of Space Debris Observations by the Virtual Debris Algorithm. In Proceedings of the Fifth European Conference on Space Debris, ESOC, Darmstadt, Germany, 30 March-2 April 2009, 2009.

[31] P. Williams, D. Spencer, and R. Erwin. Coupling of estimation and sensor tasking applied to satellite tracking. Journal of Guidance, Control and Dynamics, 36(4):993 - 1007, 2013. doi: https://doi.org/10.2514/1.59361. 